\title{
Axisymmetric Solutions to Time-Fractional Heat Conduction Equation in a Half-Space under Robin Boundary Conditions
}

\author{
Y. Z. Povstenko ${ }^{1,2}$ \\ ${ }^{1}$ Institute of Mathematics and Computer Science, Jan Dlugosz University, 42200 Czestochowa, Poland \\ ${ }^{2}$ Department of Computer Science, European University of Informatics and Economics (EWSIE), \\ 03741 Warsaw, Poland
}

Correspondence should be addressed to Y. Z. Povstenko, j.povstenko@ajd.czest.pl

Received 23 May 2012; Revised 18 July 2012; Accepted 23 July 2012

Academic Editor: Nikolai Leonenko

Copyright (C) 2012 Y. Z. Povstenko. This is an open access article distributed under the Creative Commons Attribution License, which permits unrestricted use, distribution, and reproduction in any medium, provided the original work is properly cited.

The time-fractional heat conduction equation with the Caputo derivative of the order $0<\alpha<2$ is considered in a half-space in axisymmetric case under two types of Robin boundary condition: the mathematical one with the prescribed linear combination of the values of temperature and the values of its normal derivative and the physical condition with the prescribed linear combination of the values of temperature and the values of the heat flux at the boundary.

\section{Introduction}

The generalized Fourier law, the time-nonlocal dependence between the heat flux vector $\mathbf{q}$ and the temperature gradient grad $T$ with the "long-tail memory" power kernel $[1,2]$ (see also [3]) as

$$
\begin{array}{ll}
\mathbf{q}(t)=-\frac{k}{\Gamma(\alpha)} \frac{\partial}{\partial t} \int_{0}^{t}(t-\tau)^{\alpha-1} \operatorname{grad} T(\tau) d \tau, & 0<\alpha \leq 1 \\
\mathbf{q}(t)=-\frac{k}{\Gamma(\alpha-1)} \int_{0}^{t}(t-\tau)^{\alpha-2} \operatorname{grad} T(\tau) d \tau, & 1<\alpha \leq 2
\end{array}
$$

where $\Gamma(\alpha)$ is the gamma function, can be interpreted in terms of the fractional calculus:

$$
\begin{array}{ll}
\mathbf{q}(t)=-k D_{R L}^{1-\alpha} \operatorname{grad} T(t), & 0<\alpha \leq 1, \\
\mathbf{q}(t)=-k I^{\alpha-1} \operatorname{grad} T(t), & 1<\alpha \leq 2,
\end{array}
$$


and in combination with the law of conservation of energy as

$$
\rho_{m} C \frac{d T}{d t}=-\operatorname{div} \mathbf{q}
$$

results in the time-fractional heat conduction equation with the Caputo fractional derivative

$$
\frac{\partial^{\alpha} T}{\partial t^{\alpha}}=a \Delta T, \quad 0<\alpha \leq 2
$$

Here, $\rho_{m}$ is the mass density, $C$ denotes the specific heat capacity, and $a=k /\left(\rho_{m} C\right)$ is the thermal diffusivity coefficient.

Recall that the Riemann-Liouville fractional integral $I^{\alpha} f(t)$ and derivative $D_{R L}^{\alpha} f(t)$ are defined as follows (see [4-6]):

$$
\begin{gathered}
I^{\alpha} f(t)=\frac{1}{\Gamma(\alpha)} \int_{0}^{t}(t-\tau)^{\alpha-1} f(\tau) d \tau, \quad \alpha>0, \\
D_{R L}^{\alpha} f(t)=\frac{d^{m}}{d t^{m}}\left[\frac{1}{\Gamma(m-\alpha)} \int_{0}^{t}(t-\tau)^{m-\alpha-1} f(\tau) d \tau\right], \quad m-1<\alpha<m,
\end{gathered}
$$

whereas the Caputo fractional derivative has the following form [5-7]:

$$
\frac{d^{\alpha} f(t)}{d t^{\alpha}}=\frac{1}{\Gamma(m-\alpha)} \int_{0}^{t}(t-\tau)^{m-\alpha-1} \frac{d^{m} f(\tau)}{d \tau^{m}} d \tau, \quad m-1<\alpha<m .
$$

A detailed explanation of derivation of time-fractional heat conduction equation (1.4) from the constitutive equations (1.2) and the law of conservation of energy (1.3) can be found in [8]. Here we briefly present the main idea. In the case $0<\alpha \leq 1$, as a consequence of (1.2), (1.3), and (1.6), we have

$$
\frac{\partial T}{\partial t}=a \frac{\partial}{\partial t}\left[\frac{1}{\Gamma(\alpha)} \int_{0}^{t}(t-\tau)^{\alpha-1} \Delta T(\tau) d \tau\right]
$$

or after integration with respect to time as

$$
T(t)-T(0)=a I^{\alpha} \Delta T
$$

Applying to both sides of (1.9) the Caputo derivative $\partial^{\alpha} / \partial t^{\alpha}$, and taking into account that for $\alpha>0[6]$,

$$
\frac{\partial^{\alpha}}{\partial t^{\alpha}} I^{\alpha} T(t)=T(t)
$$

we obtain the time-fractional heat conduction equation (1.4) for $0<\alpha \leq 1$. 
Similarly, for $1<\alpha \leq 2$, we get

$$
\frac{\partial T}{\partial t}=a I^{\alpha-1} \Delta T
$$

Applying $\partial^{\alpha-1} / \partial t^{\alpha-1}$ to both sides of (1.11) gives (1.4) for $1<\alpha \leq 2$ as the following equality fulfills [5]

$$
\frac{\partial^{\alpha-1}}{\partial t^{\alpha-1}} \frac{\partial T}{\partial t}=\frac{\partial^{\alpha} T}{\partial t^{\alpha}}
$$

If the heat conduction equation is investigated in a bounded domain, the boundary conditions should be prescribed. The mathematical Robin boundary condition is a specification of a linear combination of the values of temperature and the values of its normal derivative at the boundary of the considered domain

$$
\left.\left(c_{1} T+c_{2} \frac{\partial T}{\partial n}\right)\right|_{S}=F_{0}\left(\mathbf{x}_{S}, t\right)
$$

with some nonzero constants $c_{1}$ and $c_{2}$, while the physical Robin boundary condition specifies a linear combination of the values of temperature and the values of the heat flux at the boundary of the domain. For example, the condition of convective heat exchange between a body and the environment with the temperature $T_{e}$

$$
\left.\mathbf{q} \cdot \mathbf{n}\right|_{S}=h\left(\left.T\right|_{S}-T_{e}\right)
$$

where $h$ is the convective heat transfer coefficient, leads to

$$
\begin{aligned}
& \left.\left(h T+k D_{R L}^{1-\alpha} \frac{\partial T}{\partial n}\right)\right|_{S}=h T_{e}\left(\mathbf{x}_{S}, t\right), \quad 0<\alpha \leq 1, \\
& \left.\left(h T+k I^{\alpha-1} \frac{\partial T}{\partial n}\right)\right|_{S}=h T_{e}\left(\mathbf{x}_{S}, t\right), \quad 1<\alpha \leq 2 .
\end{aligned}
$$

The literature on mathematical aspects concerning correctness of initial-boundaryvalue problems for time-fractional diffusion equation and form and properties of its solutions is quite extensive (see, e.g., [9-16], among others). Geometrical explanation of fractional calculus is given in [17-19].

There are only a few papers $[20,21]$ in which the fractional diffusion is investigated under the mathematical Robin boundary condition. In previous publications, problems for a cylinder [22] and a sphere [23] under mathematical and physical Neumann boundary conditions were considered. In the present paper, for the first time, the solutions to timefractional heat conduction equation in a half-space are studied under both the mathematical and physical Robin boundary conditions. The Laplace integral transform with respect to time $t$, the Hankel transform with respect to the spatial coordinate $r$, and the sin-cosFourier transforms with respect to spatial coordinate $z$ are used. The solutions under the mathematical and physical Neumann boundary conditions are obtained as particular cases. 


\section{Mathematical Preliminaries}

\subsection{Laplace Transform}

The Laplace transform is defined as

$$
\mathcal{L}\{f(t)\}=f^{*}(s)=\int_{0}^{\infty} f(t) e^{-s t} d t
$$

where $s$ is the transform variable.

The inverse Laplace transfrom is carried out according to the Fourier-Mellin formula:

$$
\mathcal{L}^{-1}\left\{f^{*}(s)\right\}=f(t)=\frac{1}{2 \pi i} \int_{c-i \infty}^{c+i \infty} f^{*}(s) e^{s t} d s, \quad t>0,
$$

where $c$ is a positive fixed number.

The Laplace transform of the Riemann-Liouville fractional integral of the order $\alpha$ is carried out according to the formula similar to the Laplace transform of $m$-fold primitive of a function as

$$
\mathcal{L}\left\{I^{\alpha} f(t)\right\}=\frac{1}{s^{\alpha}} f^{*}(s) .
$$

The Caputo derivative for its Laplace transform requires the knowledge of the initial values of the function $f\left(0^{+}\right)$and its integer derivatives $f^{(k)}\left(0^{+}\right)$of the order $k=1,2, \ldots, m-1$

$$
\mathcal{L}\left\{\frac{d^{\alpha} f(t)}{d t^{\alpha}}\right\}=s^{\alpha} f^{*}(s)-\sum_{k=0}^{m-1} f^{(k)}\left(0^{+}\right) s^{\alpha-1-k}, \quad m-1<\alpha<m,
$$

whereas the Riemann-Liouville derivative for its Laplace transform rule requires the knowledge of the initial values of the fractional integral $I^{m-\alpha} f\left(0^{+}\right)$and its derivatives of the order $k=1,2, \ldots, m-1$

$$
\mathcal{L}\left\{D_{R L}^{\alpha} f(t)\right\}=s^{\alpha} f^{*}(s)-\sum_{k=0}^{m-1} D^{k} I^{m-\alpha} f\left(0^{+}\right) s^{m-1-k}, \quad m-1<\alpha<m
$$

The reader interested in applications of integral transforms in fractional calculus is referred to $[4-7,24]$. 


\subsection{Hankel Transform}

The Hankel transform is used to solve problem in cylindrical coordinates in the domain $0 \leq$ $r<\infty$ and is defined as

$$
\begin{gathered}
\mathscr{L}\{f(r)\}=f^{*}(\eta)=\int_{0}^{\infty} f(r) J_{v}(\eta r) r d r \\
\mathscr{L}^{-1}\left\{f^{*}(\eta)\right\}=f(r)=\int_{0}^{\infty} f^{*}(\eta) J_{v}(\eta r) \eta d \eta,
\end{gathered}
$$

where $J_{v}(r)$ is the Bessel function of the order $v$.

The following formula is fulfilled:

$$
\mathscr{H}\left\{\frac{d^{2} f(r)}{d r^{2}}+\frac{1}{r} \frac{d f(r)}{d r}-\frac{v^{2}}{r^{2}} f(r)\right\}=-\eta^{2} f^{*}(\eta)
$$

\subsection{Sin-Cos-Fourier Transform}

In the case of boundary condition of the third kind with the prescribed boundary value of linear combination of a function and its normal derivative

$$
z=0: \quad-\frac{d f}{d z}+H f=\varphi_{0}
$$

the following sin-cos-Fourier transform [25] is employed:

$$
\begin{gathered}
\mathcal{F}\{f(z)\}=f^{*}(\xi)=\int_{0}^{\infty} K(z, \xi) f(z) d z, \\
\mathcal{F}^{-1}\left\{f^{*}(\xi)\right\}=f(z)=\frac{2}{\pi} \int_{0}^{\infty} K(z, \xi) f^{*}(\xi) d \xi
\end{gathered}
$$

with the kernel

$$
K(z, \xi)=\frac{\xi \cos (z \xi)+H \sin (z \xi)}{\sqrt{\xi^{2}+H^{2}}} .
$$

Application of the sin-cos-Fourier transform to the second derivative of a function gives

$$
\mathcal{F}\left\{\frac{d^{2} f(z)}{d z^{2}}\right\}=-\xi^{2} f^{*}(\xi)-\left.\frac{\xi}{\sqrt{\xi^{2}+H^{2}}}\left[\frac{d f(z)}{d z}-H f(z)\right]\right|_{z=0} .
$$




\section{Solution to the Problem under Mathematical Robin Boundary Condition}

Consider the axisymmetric time-fractional heat conduction equation in cylindrical coordinates

$$
\begin{aligned}
& \frac{\partial^{\alpha} T}{\partial t^{\alpha}}=a\left(\frac{\partial^{2} T}{\partial r^{2}}+\frac{1}{r} \frac{\partial T}{\partial r}+\frac{\partial^{2} T}{\partial z^{2}}\right), \\
& 0 \leq r<\infty, \quad 0<z<\infty, \quad 0<t<\infty, \quad 0<\alpha \leq 2,
\end{aligned}
$$

with zero initial conditions

$$
\begin{gathered}
t=0: \quad T=0, \quad 0<\alpha \leq 2 \\
t=0: \quad \frac{\partial T}{\partial t}=0, \quad 1<\alpha \leq 2
\end{gathered}
$$

and the mathematical Robin boundary condition

$$
z=0: \quad H T-\frac{\partial T}{\partial z}=f(r, t)
$$

The zero conditions at infinity are also assumed

$$
\lim _{r \rightarrow \infty} T(r, z, t)=0, \quad \lim _{z \rightarrow \infty} T(r, z, t)=0 .
$$

The solution to the initial-boundary-value problem (3.1)-(3.4) can be written as

$$
T=\int_{0}^{t} \int_{0}^{\infty} f(\rho, \tau) \mathcal{G}_{m}(r, z, \rho, t-\tau) \rho d \rho d \tau
$$

where $\mathcal{G}_{m}(r, z, \rho, t)$ is the fundamental solution being the solution of the following problem:

$$
\begin{gathered}
\frac{\partial^{\alpha} \mathcal{G}_{m}}{\partial t^{\alpha}}=a\left(\frac{\partial^{2} \mathcal{G}_{m}}{\partial r^{2}}+\frac{1}{r} \frac{\partial \mathcal{G}_{m}}{\partial r}+\frac{\partial^{2} \mathcal{G}_{m}}{\partial z^{2}}\right), \\
0 \leq r<\infty, \quad 0<z<\infty, \quad 0<t<\infty, \quad 0<\alpha \leq 2, \\
t=0: \quad \mathcal{G}_{m}=0, \quad 0<\alpha \leq 2, \\
t=0: \quad \frac{\partial \mathcal{G}_{m}}{\partial t}=0, \quad 1<\alpha \leq 2, \\
z=0: \quad H \mathcal{G}_{m}-\frac{\partial \mathcal{G}_{m}}{\partial z}=\frac{1}{r} \delta(r-\rho) \delta(t) .
\end{gathered}
$$

Here, $\delta(r)$ is the Dirac delta function. 
The Laplace transform with respect to time $t$, the Hankel transform with respect to the spatial coordinate $r$, and the sin-cos-Fourier transform with respect to the spatial coordinate $z$ result in

$$
\mathcal{G}_{m}^{* * *}=\frac{a \xi J_{0}(\rho \eta)}{\sqrt{\xi^{2}+H^{2}}} \frac{1}{s^{\alpha}+a\left(\xi^{2}+\eta^{2}\right)}
$$

where each of the integral transforms is denoted by the asterisk.

Invertion of the integral transforms leads to

$$
\begin{aligned}
\mathcal{G}_{m}(r, z, \rho, t)= & \frac{2 a t^{\alpha-1}}{\pi} \iint_{0}^{\infty} \frac{\xi^{2} \cos (z \xi)+\xi H \sin (z \xi)}{\xi^{2}+H^{2}} \\
& \times E_{\alpha, \alpha}\left[-a\left(\xi^{2}+\eta^{2}\right) t^{\alpha}\right] J_{0}(r \eta) J_{0}(\rho \eta) \eta d \xi d \eta .
\end{aligned}
$$

Here, $E_{\alpha, \beta}$ is the Mittag-Leffler function in two parameters $\alpha$ and $\beta$ defined by the series representation:

$$
E_{\alpha, \beta}(z)=\sum_{m=0}^{\infty} \frac{z^{m}}{\Gamma(\alpha m+\beta)}, \quad \alpha>0, \beta>0, z \in C
$$

The essential role of the Mittag-Leffler function in fractional calculus results from the following formula for the inverse Laplace transform

$$
\mathcal{L}^{-1}\left\{\frac{s^{\alpha-\beta}}{s^{\alpha}+b}\right\}=t^{\beta-1} E_{\alpha, \beta}\left(-b t^{\alpha}\right) .
$$

Consider several particular cases of solution (3.8).

The case $H=0$ corresponds to the mathematical Neumann boundary condition with the prescribed boundary value of the normal derivative of temperature, and the solution reads

$$
\begin{aligned}
\mathcal{G}_{m}(r, z, \rho, t)= & \frac{2 a t^{\alpha-1}}{\pi} \iint_{0}^{\infty} E_{\alpha, \alpha}\left[-a\left(\xi^{2}+\eta^{2}\right) t^{\alpha}\right] \\
& \times \cos (z \xi) J_{0}(r \eta) J_{0}(\rho \eta) \eta d \xi d \eta .
\end{aligned}
$$


In the case of classical heat conduction $(\alpha=1)$, the Mittag-Leffler function:

$$
E_{1,1}\left(-x^{2}\right)=e^{-x^{2}}
$$

and taking into account the following integrals $[26,27]$ :

$$
\begin{aligned}
& \int_{0}^{\infty} x e^{-a x^{2}} J_{0}(b x) J_{0}(c x) d x=\frac{1}{2 a} \exp \left(-\frac{b^{2}+c^{2}}{4 a}\right) I_{0}\left(\frac{b c}{2 a}\right), \quad a>0, \\
& \int_{0}^{\infty} e^{-a x^{2}} \cos (c x) d x=\frac{\sqrt{\pi}}{2 \sqrt{a}} \exp \left(-\frac{c^{2}}{4 a}\right), \quad a>0, \\
& \int_{0}^{\infty} \frac{1}{x^{2}+b^{2}} e^{-a x^{2}} \cos (c x) d x \\
& =\frac{\pi}{4 b} e^{a b^{2}}\left[e^{-b c} \operatorname{erfc}\left(\sqrt{a} b-\frac{c}{2 \sqrt{a}}\right)+e^{b c} \operatorname{erfc}\left(\sqrt{a} b+\frac{c}{2 \sqrt{a}}\right)\right], \quad a>0, b>0, \\
& \int_{0}^{\infty} \frac{x}{x^{2}+b^{2}} e^{-a x^{2}} \sin (c x) d x \\
& =\frac{\pi}{4} e^{a b^{2}}\left[e^{-b c} \operatorname{erfc}\left(\sqrt{a} b-\frac{c}{2 \sqrt{a}}\right)-e^{b c} \operatorname{erfc}\left(\sqrt{a} b+\frac{c}{2 \sqrt{a}}\right)\right], \quad a>0, b>0,
\end{aligned}
$$

where $I_{0}(x)$ is the modified Bessel function, $\operatorname{erfc} x$ is the complementary error function, we get

$$
\begin{aligned}
\mathcal{G}_{m}(r, z, \rho, t)= & \frac{1}{2 t} \exp \left(-\frac{r^{2}+\rho^{2}+z^{2}}{4 a t}\right) I_{0}\left(\frac{r \rho}{2 a t}\right) \\
& \times\left\{\frac{1}{\sqrt{\pi a t}}-H \exp \left[\left(\sqrt{a t} H+\frac{z}{2 \sqrt{a t}}\right)^{2}\right] \operatorname{erfc}\left(\sqrt{a t} H+\frac{z}{2 \sqrt{a t}}\right)\right\} .
\end{aligned}
$$

For the wave equation $(\alpha=2)$,

$$
E_{2,2}\left(-x^{2}\right)=\frac{\sin x}{x}
$$


and after evaluation of the necessary integrals appearing in solution (3.8) (under assumption $\rho=0)[26,27]$

$$
\begin{aligned}
& \int_{0}^{\infty} \frac{x}{\sqrt{x^{2}+p^{2}}} \sin \left(b \sqrt{x^{2}+p^{2}}\right) J_{0}(c x) d x= \begin{cases}\frac{1}{\sqrt{b^{2}-c^{2}}} \cos \left(p \sqrt{b^{2}-c^{2}}\right), & 0<c<b, \\
0, & 0<b<c .\end{cases} \\
& \int_{0}^{\infty} \frac{\cos (p x) \cos (q x)}{x^{2}+b^{2}} d x=\left\{\begin{array}{lll}
\frac{\pi}{2 b} e^{-b p} \cosh (b q), & 0<q<p, & \\
\frac{\pi}{2 b} e^{-b q} \cosh (b p), & 0<p<q, & b>0,
\end{array}\right. \\
& \int_{0}^{\infty} \frac{x \cos (p x) \sin (q x)}{x^{2}+b^{2}} d x=\left\{\begin{array}{ll}
-\frac{\pi}{2} e^{-b p} \sinh (b q), & 0<q<p, \\
\frac{\pi}{2} e^{-b q} \cosh (b p), & 0<p<q,
\end{array} \quad b>0,\right.
\end{aligned}
$$

we obtain the solution

$$
\begin{aligned}
& \mathcal{G}_{m}(r, z, 0, t) \\
& \quad= \begin{cases}\frac{\sqrt{a}}{\sqrt{a t^{2}-r^{2}}}\left[\delta\left(\sqrt{a t^{2}-r^{2}}-z\right)-H e^{-H\left(\sqrt{a t^{2}-r^{2}}-z\right)}\right] & 0<r<\sqrt{a} t \\
0 & 0<z<\sqrt{a t^{2}-r^{2}}, \\
0 & \sqrt{a} t<r<\infty, \sqrt{a t^{2}-r^{2}}<z<\infty\end{cases}
\end{aligned}
$$

Of particular interest is also the case $\alpha=1 / 2$ for which

$$
\begin{aligned}
& E_{1 / 2,1 / 2}(-x)=\frac{1}{\sqrt{\pi}}-x e^{x^{2}} \operatorname{erfc} x=\frac{2}{\sqrt{\pi}} \int_{0}^{\infty} e^{-u^{2}-2 x u} u d u, \quad x \geq 0, \\
& \mathcal{G}_{m}(r, z, \rho, t)=\frac{1}{2 \sqrt{\pi} t} \int_{0}^{\infty} \exp \left(-u^{2}-\frac{r^{2}+\rho^{2}+z^{2}}{8 a \sqrt{t} u}\right) I_{0}\left(\frac{r \rho}{4 a \sqrt{t} u}\right) \\
& \times\left\{\frac{1}{\sqrt{2 \pi a u} t^{1 / 4}}-H \exp \left[\left(\sqrt{2 a u} H t^{1 / 4}+\frac{z}{2 \sqrt{2 a u} t^{1 / 4}}\right)^{2}\right]\right. \\
&\left.\times \operatorname{erfc}\left(\sqrt{2 a u} H t^{1 / 4}+\frac{z}{2 \sqrt{2 a u} t^{1 / 4}}\right)\right\} d u .
\end{aligned}
$$




\section{Solution to the Problem under Physical Robin Boundary Condition}

Consider the following axisymmetric time-fractional heat conduction equation:

$$
\begin{aligned}
& \frac{\partial^{\alpha} T}{\partial t^{\alpha}}=a\left(\frac{\partial^{2} T}{\partial r^{2}}+\frac{1}{r} \frac{\partial T}{\partial r}+\frac{\partial^{2} T}{\partial z^{2}}\right) \\
& 0 \leq r<\infty, \quad 0<z<\infty, \quad 0<t<\infty, \quad 0<\alpha \leq 2
\end{aligned}
$$

under zero initial conditions

$$
\begin{gathered}
t=0: \quad T=0, \quad 0<\alpha \leq 2 \\
t=0: \quad \frac{\partial T}{\partial t}=0, \quad 1<\alpha \leq 2
\end{gathered}
$$

and the physical Robin boundary condition

$$
\begin{array}{ll}
z=0: \quad H T-D_{R L}^{1-\alpha} \frac{\partial T}{\partial z}=f(r, t), & 0<\alpha \leq 1, \\
z=0: & H T-I^{\alpha-1} \frac{\partial T}{\partial z}=f(r, t), \quad 1<\alpha \leq 2,
\end{array}
$$

where $H=h / k$.

The solution to the initial-boundary-value problem (4.1)-(4.3) has the form

$$
T=\int_{0}^{t} \int_{0}^{\infty} f(\rho, \tau) \mathcal{G}_{p}(r, z, \rho, t-\tau) \rho d \rho d \tau,
$$

where the fundamental solution $\mathcal{G}_{p}(r, z, \rho, t)$ fulfills the following equation

$$
\begin{aligned}
& \frac{\partial^{\alpha} \mathcal{G}_{p}}{\partial t^{\alpha}}=a\left(\frac{\partial^{2} \mathcal{G}_{p}}{\partial r^{2}}+\frac{1}{r} \frac{\partial \mathcal{G}_{p}}{\partial r}+\frac{\partial^{2} \mathcal{G}_{p}}{\partial z^{2}}\right) \\
& 0 \leq r<\infty, \quad 0<z<\infty, \quad 0<t<\infty, \quad 0<\alpha \leq 2,
\end{aligned}
$$

under the following conditions:

$$
\begin{gathered}
t=0: \quad \mathcal{G}_{p}=0, \quad 0<\alpha \leq 2, \\
t=0: \quad \frac{\partial \mathcal{G}_{p}}{\partial t}=0, \quad 1<\alpha \leq 2, \\
z=0: \quad H \mathcal{G}_{p}-D_{R L}^{1-\alpha} \frac{\partial \mathcal{G}_{p}}{\partial z}=\frac{1}{r} \delta(r-\rho) \delta(t), \quad 0<\alpha \leq 1, \\
z=0: \quad H \mathcal{G}_{p}-I^{\alpha-1} \frac{\partial \mathcal{G}_{p}}{\partial z}=\frac{1}{r} \delta(r-\rho) \delta(t), \quad 1<\alpha \leq 2 .
\end{gathered}
$$


International Journal of Differential Equations

The Laplace transform with respect to time $t$ leads to the following equation:

$$
s^{\alpha} \mathcal{G}_{p}^{*}=a\left(\frac{\partial^{2} \mathcal{G}_{p}^{*}}{\partial r^{2}}+\frac{1}{r} \frac{\partial \mathcal{G}_{p}^{*}}{\partial r}+\frac{\partial^{2} \mathcal{G}_{p}^{*}}{\partial z^{2}}\right), \quad 0 \leq r<\infty, 0<z<\infty, 0<\alpha \leq 2,
$$

and the following boundary condition:

$$
z=0: \quad s^{\alpha-1} H \mathcal{G}_{p}^{*}-\frac{\partial \mathcal{G}_{p}^{*}}{\partial z}=\frac{1}{r} \delta(r-\rho) s^{\alpha-1}
$$

In this case, the kernel of the sin-cos-Fourier transform with respect to the spatial coordinate $z$ depends on the Laplace transform variable $s$

$$
K(z, \xi, s)=\frac{\xi \cos (z \xi)+s^{\alpha-1} H \sin (z \xi)}{\sqrt{\xi^{2}+\left(s^{\alpha-1} H\right)^{2}}},
$$

and in the transform domain we obtain

$$
\mathcal{G}_{p}^{* * *}=\frac{a \xi J_{0}(\rho \eta)}{\sqrt{\xi^{2}+\left(s^{\alpha-1} H\right)^{2}}} \frac{s^{\alpha-1}}{s^{\alpha}+a\left(\xi^{2}+\eta^{2}\right)} .
$$

Inversion of the Laplace transform in (4.10) depends on the value of $\alpha$. For $0<\alpha \leq 1$, we have

$$
\begin{aligned}
\mathcal{G}_{p}(r, z, \rho, t)= & \frac{2 a}{\pi} \iint_{0}^{\infty} \int_{0}^{t} \eta J_{0}(r \eta) J_{0}(\rho \eta) \tau^{\alpha-1} E_{\alpha, \alpha}\left[-a\left(\xi^{2}+\eta^{2}\right) \tau^{\alpha}\right] \\
\times & \left\{(t-\tau)^{-\alpha} E_{2-2 \alpha, 1-\alpha}\left[-\frac{H^{2}}{\xi^{2}}(t-\tau)^{2-2 \alpha}\right] \cos (z \xi)\right. \\
& \left.+\frac{H}{\xi}(t-\tau)^{1-2 \alpha} E_{2-2 \alpha, 2-2 \alpha}\left[-\frac{H^{2}}{\xi^{2}}(t-\tau)^{2-2 \alpha}\right] \sin (z \xi)\right\} d \tau d \xi d \eta,
\end{aligned}
$$

whereas for $1<\alpha \leq 2$, we get

$$
\begin{aligned}
\mathcal{G}_{p}(r, z, \rho, t)= & \frac{2 a}{\pi} \iint_{0}^{\infty} \int_{0}^{t} J_{0}(r \eta) J_{0}(\rho \eta) E_{\alpha}\left[-a\left(\xi^{2}+\eta^{2}\right) \tau^{\alpha}\right] \\
& \times\left\{\frac{\xi^{2}}{H^{2}}(t-\tau)^{2 \alpha-3} E_{2 \alpha-2,2 \alpha-2}\left[-\frac{\xi^{2}}{H^{2}}(t-\tau)^{2 \alpha-2}\right] \cos (z \xi)\right. \\
& \left.+\frac{\xi}{H}(t-\tau)^{\alpha-2} E_{2 \alpha-2, \alpha-1}\left[-\frac{H^{2}}{\xi^{2}}(t-\tau)^{2-2 \alpha}\right] \sin (z \xi)\right\} d \tau d \xi d \eta .
\end{aligned}
$$


The fundamental solution under physical Neumann boundary condition is obtained for $H=$ 0 as

$$
\mathcal{G}_{p}(r, z, \rho, t)=\frac{2 a}{\pi} \iint_{0}^{\infty} E_{\alpha}\left[-a\left(\xi^{2}+\eta^{2}\right) t^{\alpha}\right] J_{0}(r \eta) J_{0}(\rho \eta) \eta \cos (z \xi) d \xi d \eta
$$

Formulae (4.11) and (4.12) simplify for $\alpha=1 / 2$ and $\alpha=3 / 2$, respectively, taking into account (3.12) and that

$$
E_{1,1 / 2}\left(-x^{2}\right)=\frac{1}{\sqrt{\pi}}[1-2 x D(x)]
$$

where

$$
D(x)=e^{-x^{2}} \int_{0}^{x} e^{u^{2}} d u
$$

is the Dawson integral.

\section{Conclusion}

We have derived the analytical solutions to time-fractional heat conduction equation in a halfspace under mathematical and physical Robin boundary conditions. The integral transform technique has been used. It should be emphasized that in the case of physical Robin boundary condition, the order of integral transforms is important as the kernel of the sin-cos-Fourier transform depends on the Laplace transform variable. The limiting case $H=0$ corresponds to solutions of problems under mathematical and physical Neumann boundary conditions with the prescribed boundary value of the normal derivative and with the prescribed boundary value of the heat flux, respectively. The difference between mathematical and physical boundary conditions (as well as the difference between the solutions) disappears in the case of standard heat conduction equation $(\alpha=1)$.

\section{References}

[1] Y. Z. Povstenko, "Fractional heat conduction equation and associated thermal stress," Journal of Thermal Stresses, vol. 28, no. 1, pp. 83-102, 2005.

[2] Y. Z. Povstenko, "Theory of thermoelasticity based on the space-time-fractional heat conduction equation," Physica Scripta, vol. T136, Article ID 014017, 6 pages, 2009.

[3] R. Gorenflo, F. Mainardi, D. Moretti, and P. Paradisi, "Time fractional diffusion: a discrete random walk approach," Nonlinear Dynamics, vol. 29, no. 1-4, pp. 129-143, 2002.

[4] S. G. Samko, A. A. Kilbas, and O. I. Marichev, Fractional Integrals and Derivatives, Gordon and Breach Science Publishers, Yverdon, Switzerland, 1993.

[5] I. Podlubny, Fractional Differential Equations, vol. 198 of Mathematics in Science and Engineering, Academic Press, San Diego, Calif, USA, 1999.

[6] A. A. Kilbas, H. M. Srivastava, and J. J. Trujillo, Theory and Applications of Fractional Differential Equations, vol. 204 of North-Holland Mathematics Studies, Elsevier Science, Amsterdam, The Netherlands, 2006. 
[7] R. Gorenflo and F. Mainardi, "Fractional calculus: integral and differential equations of fractional order," in Fractals and Fractional Calculus in Continuum Mechanics, A. Carpinteri and F. Mainardi, Eds., vol. 378 of CISM Courses and Lectures, pp. 223-276, Springer, Vienna, 1997.

[8] Y. Povstenko, "Non-axisymmetric solutions to time-fractional diffusion-wave equation in an infinite cylinder," Fractional Calculus and Applied Analysis, vol. 14, no. 3, pp. 418-435, 2011.

[9] W. Wyss, "The fractional diffusion equation," Journal of Mathematical Physics, vol. 27, no. 11, pp. 27822785, 1986.

[10] W. R. Schneider and W. Wyss, "Fractional diffusion and wave equations," Journal of Mathematical Physics, vol. 30, no. 1, pp. 134-144, 1989.

[11] F. Mainardi, "The fundamental solutions for the fractional diffusion-wave equation," Applied Mathematics Letters, vol. 9, no. 6, pp. 23-28, 1996.

[12] F. Mainardi, "Fractional relaxation-oscillation and fractional diffusion-wave phenomena," Chaos, Solitons and Fractals, vol. 7, no. 9, pp. 1461-1477, 1996.

[13] R. Gorenflo and F. Mainardi, "Signalling problem and Dirichlet-Neumann map for time-fractional diffusion-wave equations," Matimyás Matematika, vol. 21, pp. 109-118, 1998.

[14] A. Hanyga, "Multidimensional solutions of time-fractional diffusion-wave equations," Proceedings of the Royal Society A, vol. 458, no. 2020, pp. 933-957, 2002.

[15] S. D. Eidelman and A. N. Kochubei, "Cauchy problem for fractional diffusion equations," Journal of Differential Equations, vol. 199, no. 2, pp. 211-255, 2004.

[16] Y. Luchko, "Some uniqueness and existence results for the initial-boundary-value problems for the generalized time-fractional diffusion equation," Computers $\mathcal{E}$ Mathematics with Applications, vol. 59, no. 5, pp. 1766-1772, 2010.

[17] J.-H. He, "Approximate analytical solution for seepage flow with fractional derivatives in porous media," Computer Methods in Applied Mechanics and Engineering, vol. 167, no. 1-2, pp. 57-68, 1998.

[18] J.-H. He, "A short remark on fractional variational iteration method," Physics Letters A, vol. 375, no. 38, pp. 3362-3364, 2011.

[19] J.-H. He, S. K. Elagan, and Z. B. Li, "Geometrical explanation of the fractional complex transform and derivative chain rule for fractional calculus," Physics Letters A, vol. 376, no. 4, pp. 257-259, 2012.

[20] A. K. Bazzaev and M. Kh. Shkhanukov-Lafishev, "Locally one-dimensional scheme for fractional diffusion equation with Robin boundary conditions," Computational Mathematics and Mathematical Physics, vol. 50, no. 7, pp. 1141-1149, 2010.

[21] J. Kemppainen, "Existence and uniqueness of the solution for a time-fractional diffusion equation with Robin boundary condition," Abstract and Applied Analysis, vol. 2011, Article ID 321903, 11 pages, 2011.

[22] Y. Povstenko, "Time-fractional radial heat conduction in a cylinder and associated thermal stresses," Archive of Applied Mechanics, vol. 82, no. 3, pp. 345-362, 2012.

[23] Y. Z. Povstenko, "Central symmetric solution to the Neumann problem for a time-fractional diffusionwave equation in a sphere," Nonlinear Analysis: Real World Applications, vol. 13, no. 3, pp. 1229-1238, 2012.

[24] X. Yang, "Local fractional integral transforms," Progress in Nonlinear Science, vol. 4, pp. 1-225, 2011.

[25] A. S. Galitsyn and A. N. Zhovsky, Integral Transforms and Special Functions in Heat Conduction Problems, Naukova Dumka, Kiev, Ukraine, 1976 (Russian).

[26] A. P. Prudnikov, Yu. A. Brychkov, and O. I. Marichev, Integrals and Series. Special Functions, Nauka, Moscow, Russia, 1983 (Russian).

[27] A. Erdélyi, W. Magnus, F. Oberhettinger, and F. Tricomi, Tables of Integral Transforms, vol. 1, McGrawHill, New York, NY, USA, 1954. 


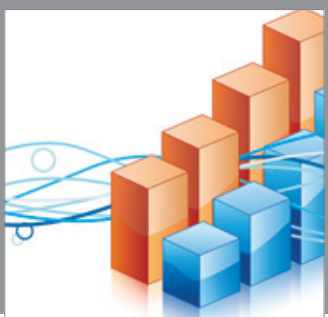

Advances in

Operations Research

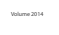

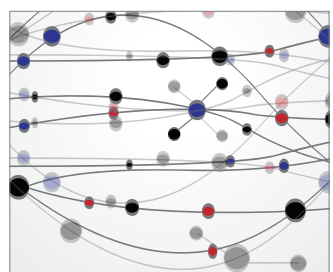

\section{The Scientific} World Journal
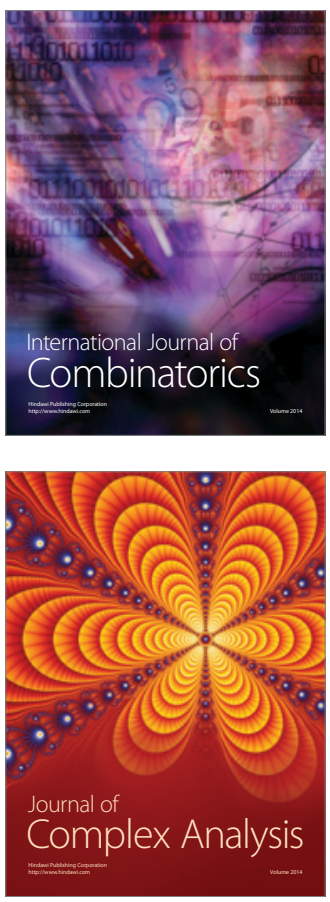

International Journal of

Mathematics and

Mathematical

Sciences
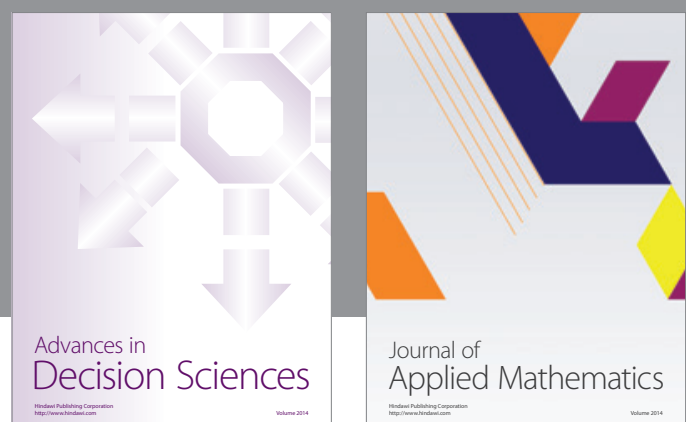

Journal of

Applied Mathematics
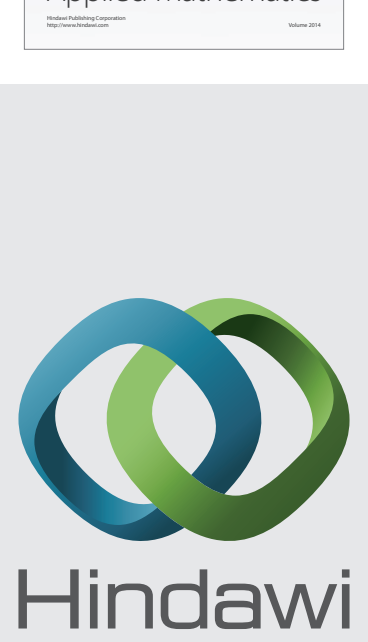

Submit your manuscripts at http://www.hindawi.com
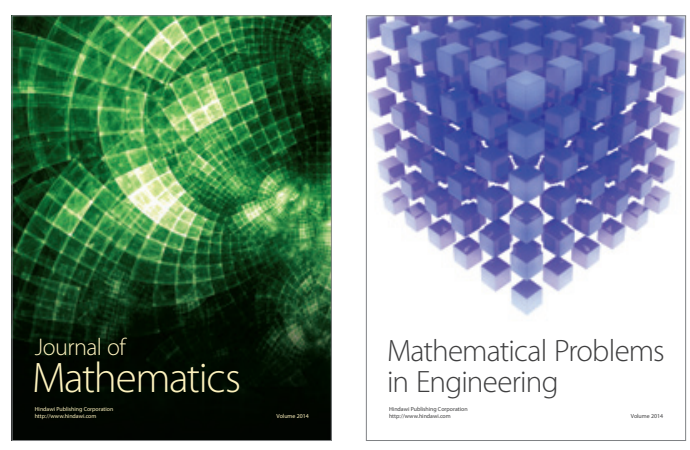

Mathematical Problems in Engineering
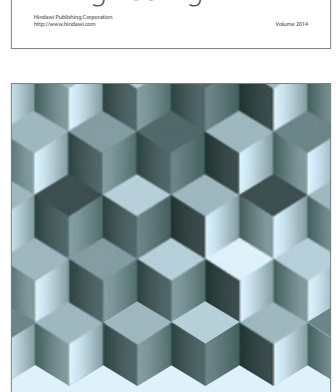

Journal of

Function Spaces
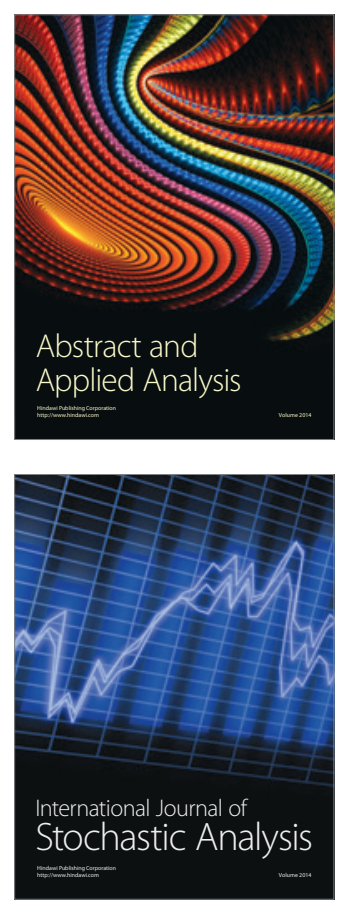

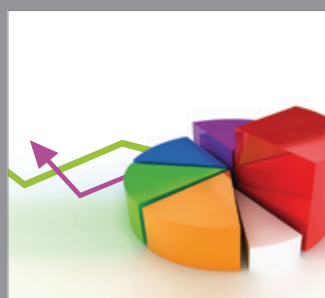

ournal of

Probability and Statistics

Promensencen
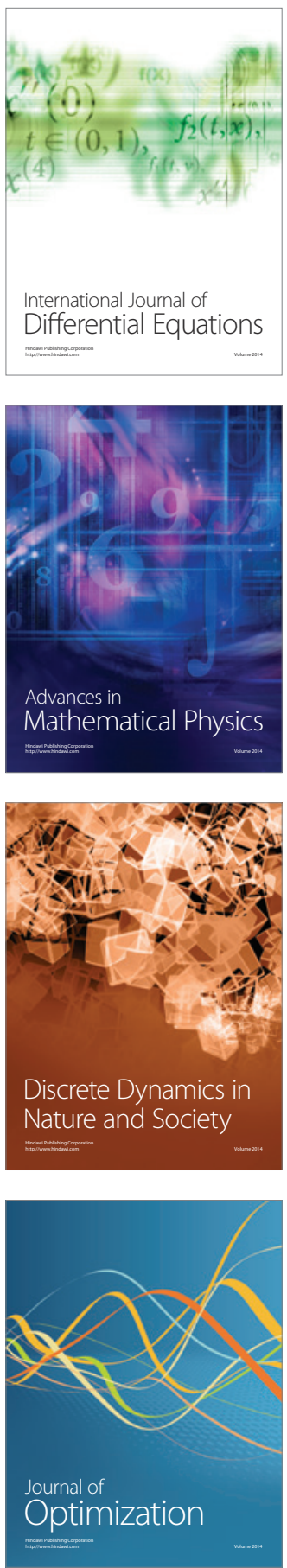